\title{
ORIGINAL
}

\section{FACTORES ASOCIADOS A MAL ESTADO DE SALUD PERCIBIDO O A MALA CALIDAD DE VIDA EN PERSONAS MAYORES DE 65 AÑOS (*)}

Mercedes Azpiazu Garrido (1), Alfonso Cruz Jentoft (2), José Ramón Villagrasa Ferrer (3), Juan Carlos Abanades Herranz (4), Natividad García Marín (5), Fernando Alvear Valero de Bernabé (6)

(1) Gerencia de Atención Primaria de Talavera de la Reina

(2) Hospital Ramón y Cajal, Madrid

(3) Hospital la Princesa, Madrid

(4) Gerencia de Atención Primaria - Área 4, Madrid

(5) Centro de Salud Pública del Área 1, Árganda, Madrid

(6) Centro de Salud Jaime Vera. Coslada - Madrid

(*) Trabajo financiado mediante el proyecto del Fondo de Investigaciones Sanitarias 99/0248

\section{RESUMEN}

Fundamento: Hoy en día, en los países desarrollados, llegar a una edad avanzada ha dejado de ser algo excepcional, sin embargo muchas personas no logran envejecer con una buena calidad de vida. El presente trabajo tiene como objetivos, contribuir a un mejor conocimiento de los principales factores que influyen en la calidad de vida y la percepción de la salud de los mayores de 65 años, así como determinar qué ventajas y desventajas presenta cada uno de los tres instrumentos de medida de la salud y la calidad de vida frente a los otros dos instrumentos utilizados.

Métodos: A partir de 911 encuestas a personas mayores de 65 años no institucionalizadas, realizadas a domicilio, se hizo un análisis multivariante mediante regresión logística, poniendo en relación los resultados obtenidos en el Perfil de Salud de Nottingham (PSN), el Euroqol y el EAS con las caracterísiticas sociodemográficas, el nivel de recursos económicos, el grado de apoyo sociofamiliar, el estado de salud física y mental y la capacidad funcional.

Resultados: Los principales factores que se asocian con la percepción de un mal estado de salud y mala calidad de vida en el Euroqol y el PSN son los trastornos de ansiedad (Odd Ratios entre 1,8 (IC:1,2-2,8) para movilidad y 7,9 (IC:4,5-13,9) para Perfil $\neq 11111$ ), trastornos depresivos (OR:1,8 (IC:1,3-2,6) para dolor/malestar $-3,3$ (IC:2,1-5,1) para aislamiento social), falta de ejercicio (OR:1,4 (IC:1-2,1) para ansiedad/depresión -3,9 (IC:2,5-6,2) para actividades cotidianas), dependencia para las actividades básicas de la vida diaria (OR:0,5 (IC:0,3-0,9) para reacción emocional $-4,8$ (IC:3-7,6) para actividades cotidianas) y dependencia para las actividades instrumentales de la vida diaria (OR:1,5 (IC:1,1-2,1) para Escala Visual Analógicac $<70-7,1$ (IC:2,9-17,2) para cuidado personal).

Conclusiones: La salud mental y la capacidad funcional son los factores que más influyen en la percepción del estado de salud y la calidad de vida de las personas mayores. Dado que los tres instrumentos utilizados han obtenido resultados semejantes, el Euroqol ofrece ventajas por su brevedad, incluyendo una valoración global y por dimensiones.

Palabras clave: Calidad de vida. Anciano. Estado de salud. Euroqol. Perfil de Salud.

Correspondencia:

Mercedes Azpiazu Garrido

C/ Guzmán el Bueno $63,4 .^{\circ} \mathrm{E}$

28015 Madrid

Correo electrónico: mazpiazu@sescam.org

\section{ABSTRACT}

\section{Factors Related to Perceived Poor Health Condition or Poor Quality of Life among those over Age 65}

Background: Nowadays, in the developed countries, a long lifespan is no longer the exception to the rule, however there are still many people who even today do not manage to age with a good quality of life. The objectives of this study are, first of all, to contribute to a better knowledge of the main factors which have an impact on the quality of life and the perceived health condition of those over age 65 and, secondly, to determine what advantages and disadvantages involved in each one of the tools for gauging health and quality of life as compared to the other two tools employed.

Methods: Based on 911 home surveys of non-institutionalized individuals over age 65 , a multivariate analysis was made using Logistic regression, relating the results obtained in the Nottingham Health Profile (NHP), the EuroQol and the Self Perceived Health Status to the socio-demographic characteristics, the level of economic resources, the degree of social-family support, the physical and mental health condition and the functional capacity.

Results: The main factors related to the perception of a poor health condition and a poor quality of life in the EuroQol and the NHP are anxiety disorders (Odds Ratio ranging from 1.8(IC:1.2-2.8) for mobility and 7.9(IC:4.5-13.9) for Profile*11111), depressive disorders (OR:1.8(IC:1.3-2.6) for pain/discomfort- 3.3(IC:2.1-5.1) for social isolation), lack of exercise (OR:1.4 (IC:1-2.1) for anxiety/depression -3.9(IC:2.5-6.2) for everyday activities), dependence for basic everyday living activities (OR:0.5(IC:0.3-0.9) for emotional reaction - 4.8(IC:3-7.6) for everyday activities) and dependence for the instrumental daily living activities (OR:1.5(IC:1.1-2.1) for Analog Visual Scale $\mathrm{c}<70$ - 7.1(IC:2.9-17.2) for personal care).

Conclusions: Mental health and functioning capacity are the factors which have the greatest bearing on the perception of health condition and quality of life of individuals over age 65 Given that the three tools used have led to similar results, the EuroQol has advantages to offer due to its short length, including an overall evaluation by dimensions.

Key words: Quality of life, Elderly, Health status, Euroqol, Nottingham Health Profile. 


\section{INTRODUCCIÓN}

Hoy en día, en los países desarrollados, llegar a una edad avanzada ha dejado de ser algo excepcional, sin embargo aún hoy muchas personas no logran envejecer con una buena calidad de vida. La calidad de vida de una persona resulta de la interacción de múltiples factores (socioeconómicos, salud mental, salud física, función social...). Badia y cols ${ }^{1}$ definen la calidad de vida como la percepción global de satisfacción en un determinado número de dimensiones clave, con especial énfasis en el bienestar del individuo. este término presenta distintos significados para distintas personas, pues el patrón con el que un individuo valora su propia calidad de vida depende de su experiencia y de la distancia que exista entre sus expectativas y sus logros 2 .

El estado de salud de las personas es uno de los principales determinantes de su calidad de vida, pero también es un constructo complejo en el que a su vez influyen diversos factores. Jonhson y Wolinsky (1993) ${ }^{3}$ elaboran un modelo en el que la enfermedad produce un deterioro físico que conduce a una limitación para realizar actividades o comportamientos habituales, lo cual repercutirá en la percepción del propio estado de salud. Cada una de las fases de este modelo puede tener también un efecto directo sobre fases posteriores no inmediatas. Por ejemplo, el mero conocimiento de padecer una enfermedad, independientemente del deterioro físico que produzca, también puede dar lugar a un cambio en las actividades o comportamientos de la persona y a un empeoramiento en la percepción de su salud. Los factores sociodemográficos tienen un efecto modulador en cada una de estas fases. Así, las personas de mayor edad con un mismo grado de problemas de salud se perciben como más sanas que las de menor edad.

Verbrugge y cols ${ }^{4}$ plantean un modelo para explicar el proceso que conduce desde la enfermedad hasta la discapacidad o difi- cultad que una persona enferma tiene para desempeñar su rol social, siendo el grado de discapacidad uno de los factores que influyen sobre la calidad de vida de las personas y dependiendo éste de la interrelación entre la persona y su ambiente. La discapacidad para una determinada actividad ocurre cuando la capacidad personal para esa actividad no puede satisfacer la demanda del ambiente, de modo que la discapacidad puede disminuir tanto aumentando la capacidad como disminuyendo la demanda.

En las personas mayores la calidad de vida está principalmente determinada por la compleja interacción entre las características del individuo y su ambiente ${ }^{5}$. Las políticas de salud diseñadas en la última década para responder a las necesidades de las personas de edad avanzada buscan el mantenimiento de la capacidad del mayor para llevar una vida independiente en la comunidad con un alojamiento y un soporte social adecuados ${ }^{6-11}$. En las personas de edad el nivel de salud y de bienestar parece estar estrechamente ligado al nivel de protección social ofertado, ya que sufren muchos problemas crónicos y discapacidades, consumiendo gran cantidad de recursos sanitarios y sociales, de forma directamente proporcional al grado de dependencia ${ }^{12}$. La autonomía de los ancianos es un componente importante de su calidad de vida, de modo que el enfoque asistencial de la ancianidad debería dirigirse a la satisfacción de sus demandas y a la búsqueda de déficits encubiertos, con la instauración de las medidas correctoras pertinentes, incluyendo actividades de promoción y prevención ${ }^{13}$. Algunos estudios como «Envejecer en Leganés» ${ }^{14}$ procuran esclarecer la relación existente entre las incapacidades funcionales de las personas mayores, el apoyo social de que disponen y la utilización de los servicios sanitarios y sociales.

Diversos autores han investigado sobre los factores de la vida que son considerados importantes para la calidad de vida por las personas de edad, encontrando como varia- 
bles más importantes las relaciones familiares y contactos sociales, la salud general, el estado funcional, la vivienda y la disponibilidad económica ${ }^{15-17}$. Son componentes muy importantes de la calidad de vida la capacidad de control y las oportunidades potenciales que la persona tenga a lo largo de su vi$\mathrm{da}^{18,19}$, de forma que la calidad de vida viene dada por el grado de satisfacción alcanzado por la persona con las oportunidades que le ofrece la vida y lo conseguido en aquellos aspectos que tienen importancia para ella. Lo que los mayores necesitan para disfrutar de la vida es ser productivos y contribuir activamente en la vida de algún modo ${ }^{20}$. El crecimiento personal, el tener un propósito y una meta, y la calidad de las relaciones personales son parte de un funcionamiento positivo en la edad avanzada. Las aspiraciones de autodesarrollo y el interés en el bienestar de los demás se asocian con sentimientos de bienestar en la vejez ${ }^{21}$.

El conocimiento de qué factores son más relevantes para la población mayor de 65 años en la percepción de la calidad de su vida es fundamental para diseñar estrategias que la mejoren en este sector de la población, cada día más numeroso. Por otra parte los instrumentos de medida de la calidad de vida pueden ser de gran utilidad tanto para la valoración individual del paciente mayor en la práctica clínica como para la valoración de este grupo de población, con el fin de determinar sus necesidades de salud y sus preferencias como ayuda para la planificación de servicios sanitarios y sociales $^{22,23}$.

El presente trabajo tiene como objetivos, en primer lugar contribuir a un mejor conocimiento de los principales factores que influyen en la calidad de vida y la percepción de la salud de los mayores de 65 años, y en segundo lugar determinar qué ventajas $y$ desventajas presenta cada uno de los tres instrumentos de medida de la salud y la calidad de vida frente a los otros dos instrumentos utilizados.

\section{SUJETOS Y MÉTODOS}

Para este artículo se han analizado una parte de las variables de un estudio descriptivo transversal más amplio ${ }^{24}$. La información se ha obtenido mediante una encuesta estructurada administrada a domicilio por entrevistadores previamente entrenados, a 911 personas mayores de 65 años no institucionalizadas y que residían en las áreas sanitarias 2 y 4 de la Comunidad de Madrid. El cuestionario se compone de preguntas ampliamente utilizadas en otros cuestionarios y en la valoración clínica y social del anciano junto con test previamente validados por otros autores.

Las personas de la muestra (junto con dos sustitutos por cada una) fueron seleccionadas por muestreo sistemático con arranque aleatorio del padrón municipal. El trabajo de campo se realizó entre junio de 1999 y abril de 2000 .

Se excluyeron, aparte de las personas fallecidas y de los errores del padrón, a las personas que habían trasladado su domicilio fuera de las dos áreas sanitarias de estudio, vivían en una residencia o estaban hospitalizadas en el momento del trabajo de campo, presentaban deterioro cognitivo moderado o severo (puntuación en el test de Pfeiffer superior a 4 y las que eran incapaces de comunicarse con el entrevistador por diversas razones (sordera extrema, idioma, disfasia, disartria...).

Para intentar reducir las negativas a colaborar en el estudio se enviaron cartas en papel oficial del Insalud a las personas seleccionadas para pedirles su colaboración y se informó a los centros de salud de la zona.

Se excluyó del estudio, siendo reemplazados por otras personas, a aquellos individuos que no pudieron ser localizados en su domicilio después de tres intentos de contactar con ellos a diferentes horas del día (por teléfono o acudiendo al domicilio). 
En la tabla 1 se especifican las variables utilizadas en este artículo. Se han agrupado para el análisis sus distintas categorías, de forma que todas las variables quedan como dicotómicas y por lo tanto los resultados resultan más fáciles de entender. Se ha considerado que no tenían estudios las personas analfabetas o que sólo sabían leer y escribir. Para la asignación de clase social, se preguntó la profesión del cabeza de familia, haciéndose corresponder de la siguiente forma: Clases I o II (Autónomo o empresario con 5 empleados o menos, Empresario con 6 empleados o más, Profesional por cuenta propia o ajena, Miembro de la dirección, Responsable de 5 subordinados o menos, Responsable de 6 subordinados o más); Clase III (Mandos intermedios, Otros empleados con trabajo de oficina, Otros empleados con trabajo fuera de oficina); Clase IV o V (Obrero cualificado, Trabajador manual no cualificado, Pensionista) y Agricultor (Agricultor de pequeña explotación, Agricultor de gran explotación). Para investigar los recursos económicos y el apoyo sociofamiliar se hicieron preguntas de valoración subjetiva a la persona encuestada.

La valoración del Estado Autopercibido de Salud (EAS) se hizo con la pregunta de la Encuesta Nacional de Salud ${ }^{25}$ : ¿Cómo describiría su estado de salud en la actualidad? Muy bueno, Bueno, Regular, Malo y Muy malo. La variable hospitalización se refiere al ingreso en un hospital durante el último año. Para la valoración de la vista y oído se hicieron las preguntas $¿$ Tiene Ud. dificultades para ver? y ¿Tiene Ud. dificultad para oír una conversación normal? Se preguntó a las personas entrevistadas si hacían algo de ejercicio habitualmente.

Se valoró la capacidad funcional mediante la dimensión Actividades de la Vida Diaria del cuestionario OARS-MFAQ ${ }^{26}$ que consta de 7 ítems referentes a las actividades básicas o dirigidas al cuidado personal (AVD-B) y otros 7 ítems referentes a actividades instrumentales o de mantenimiento del medio ambiente (AVD-I). Se consideran independientes para las AVD-I o para las
AVD-B las personas capaces de realizar los 7 ítems incluidos en cada una de ellas, dependientes parciales o necesitan ayuda cuando requieren ayuda para al menos un ítem y dependientes totales cuando no pueden hacer al menos un ítem.

La existencia de trastornos afectivos se investigó a través de la escala Hospitalaria de Ansiedad y Depresión ${ }^{26}$ (HAD), que es un cuestionario formado por 14 ítems divididos en las dos subescalas Ansiedad (HAD-A) y Depresión (HAD-D), cada una de las cuales consta de 7 ítems que se evalúan en una escala de tipo Likert con puntuaciones de 0 a 3, correspondiendo la puntuación 0 a la respuesta más favorable y 3 a la respuesta menos favorable. Se obtiene una puntuación para cada subescala, considerándose las puntuaciones iguales o superiores a 11 como indicativas de caso de trastorno depresivo o de ansiedad, no caso cuando la puntuación es igual o inferior a 7, y caso dudoso con puntuaciones comprendidas entre 8 y 10 .

El Euroqol ${ }^{26}$ (EQ) y el Perfil de Salud de Nottingham $^{26}$ (PSN) son cuestionarios genéricos de Calidad de Vida Relacionada con la Salud. El EQ describe el estado de salud con cinco dimensiones (movilidad, cuidado personal, actividades cotidianas, dolor/malestar, y ansiedad/depresión), cada una de las cuales se define con tres niveles de gravedad. También consta de una Escala Visual Analógica (EVA) milimetrada en la que el sujeto debe puntuar su estado de salud en el día de la encuesta, siendo los extremos de la escala el peor estado de salud imaginable (0) y el mejor estado de salud imaginable (100). El PSN consta de una serie de ítems a los que se contesta Sí o No y que se agrupan para formar seis dimensiones (energía, dolor, movilidad física, reacciones emocionales, sueño y aislamiento social). Cada dimensión se puntúa de 0 a 100 , siendo tanto más alta la puntuación cuanto mayores sean los problemas con ella.

Al igual que otros autores ${ }^{27-29}$ se consideró mal estado percibido de salud cuando 
Tabla 1

Variables del estudio y descripción de la muestra

\begin{tabular}{|c|c|c|c|}
\hline & & $\%$ & I.C $95 \%$ \\
\hline \multicolumn{4}{|l|}{ Características sociodemográficas: } \\
\hline Edad & $\begin{array}{l}65-79 \text { años } \\
\geq 80 \text { años }\end{array}$ & 75,1 & $72,1-77,8$ \\
\hline \multirow{2}{*}{ Sexo } & & $\begin{array}{l}2,9 \\
40,3\end{array}$ & $37,1-43,6$ \\
\hline & Mujer & 59,7 & $56,4-62,9$ \\
\hline \multirow[t]{2}{*}{ Área } & \multirow{2}{*}{$\begin{array}{l}2 \\
4\end{array}$} & 53,2 & $49,9-56,5$ \\
\hline & & 46,8 & $43,5-50,1$ \\
\hline \multirow[t]{2}{*}{ Estado civil } & Casado & 59,8 & $56,5-63$ \\
\hline & Otros & 40,2 & $37-43,5$ \\
\hline Nivel de estudios & No estudios & 40,9 & $37,7-44,2$ \\
\hline & Otros & 59,1 & $55,8-62,3$ \\
\hline Clase social & I-II & 38,3 & $35,1-41,6$ \\
\hline & Otros & 61,7 & $58,4-64,9$ \\
\hline Recursos económicos: & & & \\
\hline Dependencia económica & Sí & 29,4 & $26,5-32,5$ \\
\hline & & 70,6 & $67,5-73,5$ \\
\hline Ingresos familiares & Insuficientes & 32,9 & $29,8-36$ \\
\hline & Justos u Holgados & 67,1 & $64-70,2$ \\
\hline Apoyo sociofamiliar: & & & \\
\hline Vive & Solo & 19,5 & $17-22,2$ \\
\hline & Acompañado & 80,5 & $77,8-83$ \\
\hline Está solo al día & Nunca ó Ratos & 71 & $67,8-74$ \\
\hline & Medio ó Todo el día & 29 & $26-32,2$ \\
\hline Dispone de Cuidador potencial & Sí & 88,6 & $86,2-90,7$ \\
\hline & No & 11,4 & $9,3-13,8$ \\
\hline Dispone de Confidente & Sí & 87,8 & $85,5-89,8$ \\
\hline & No & 12,2 & $10,2-14,5$ \\
\hline Salud física: & & & \\
\hline Estado de salud autopercibida & Muy buena ó Buena & 52,1 & $48,8-55,4$ \\
\hline & Regular, Mala ó Muy mala & 47,9 & $44,6-51,2$ \\
\hline Hospitalización & Sí & 19,2 & $16,8-22$ \\
\hline & No & 80,8 & $78-83,2$ \\
\hline Tabaquismo & Nunca ó Exfumador & 90,2 & $88-92$ \\
\hline & Fumador actual & 9,8 & $8-12$ \\
\hline Toma alcohol & Nunca ó En el pasado & 72,3 & $69,3-75,2$ \\
\hline & Actualmente & 27,7 & $24,8-30,7$ \\
\hline Vista & No dificultad ó Usa gafas & 89,2 & $86,9-91,1$ \\
\hline & Incapacidad ó Ceguera & 10,8 & $8,9-13,1$ \\
\hline Oído & No dificultad ó Poca dificultad & 86,5 & $84,1-88,6$ \\
\hline & Dificultad importante & 13,5 & $11,4-15,9$ \\
\hline Ejercicio & Paseos ó Deporte & 65,3 & $62,1-68,4$ \\
\hline & Cerca de casa ó En casa ó Cama/sillón & 34,7 & $31,6-37,9$ \\
\hline Capacidad funcional & & & \\
\hline Actividades instrumentales (OARS-MFAQ) & Independiente & 58 & $54,7-61,2$ \\
\hline & Dependiente parcial ó total & 42 & $38,8-45,3$ \\
\hline Actividades básicas (OARS-FMAQ & Independiente & 78 & $75,1-80,6$ \\
\hline & Dependiente parcial ó total & 22 & $22-24,9$ \\
\hline Salud mental: & & & \\
\hline Deterioro cognitivo: test de Pfeiffer & $0-2$ & 91,9 & $89,9-93,5$ \\
\hline & $3-4$ & 8,1 & $6,5-10,1$ \\
\hline Escala Hospitalaria de Ansiedad y Depresió & D): & & \\
\hline Subescala HAD-Ansiedad & No caso & 76,4 & $73,4-79,1$ \\
\hline & Caso dudoso ó Caso & 23,6 & $20,9-26,6$ \\
\hline Subescala HAD-Depresión & No caso & 72,1 & $69-74,9$ \\
\hline & Caso dudoso ó Caso & 27,9 & $25,1-31$ \\
\hline Calidad de vida: & & & \\
\hline Perfil de Salud de Nottingham & Problemas en Energía & 36,1 & $33-39,3$ \\
\hline & $"$ Dolor & 63,4 & $60,1-66,6$ \\
\hline & $" \quad$ Movilidad física & 74,4 & $71,4-77,2$ \\
\hline & Sueño & 62,1 & $58,9-65,3$ \\
\hline & Reacción emocional & 71,3 & $68,1-74,2$ \\
\hline & " Aislamiento social & 33,3 & $30,3-36,5$ \\
\hline Euroqol & Problemas en Movilidad & 21,3 & $18,7-24,1$ \\
\hline & " Cuidado personal & 7,7 & $6,1-9,7$ \\
\hline & Actividades cotidianas & 19,9 & $17,4-22,7$ \\
\hline & Dolor/malestar & 38,2 & $35-41,5$ \\
\hline & " Ansiedad/ Depresión & 27 & $24,1-30$ \\
\hline & VAS $<70$ & 48,8 & $45,5-52,1$ \\
\hline & Perfil $\neq 11111$ & 54,4 & $51,1-57,7$ \\
\hline
\end{tabular}


la persona encuestada contestó que su estado de salud era regular, malo o muy malo; mala calidad de vida en el $E Q^{30}$ cuando la persona encuestada presentaba algunos o muchos problemas en cualquiera de sus dimensiones, el perfil de EQ era diferente del perfil 11111 o el valor de la EVA inferior a la mediana de la muestra (70); y mala calidad de vida en el $P S N^{31}$ cuando la persona encuestada tuvo una puntuación superior a 0 en cualquiera de las dimensiones del PSN.

El análisis estadístico se realizó con el programa SPSS v 8.0. Se comprobó en un primer paso, mediante comparación de proporciones (test de $\chi^{2}$ ), la existencia de asociación de mal estado de salud percibido o mala calidad de vida con las características sociodemográficas, el nivel de recursos económicos, el grado de apoyo familiar, el estado de salud física y mental y la capacidad funcional de la población estudiada. Posteriormente se hizo análisis multivariante mediante regresión logística con el EAS y los resultados obtenidos en el EQ y el PSN como variables dependientes. Para ello se utilizó una estrategia hacia atrás, partiendo en todos los casos del modelo máximo y sacando las variables cuyo coeficiente de regresión tenía una $\mathrm{p}>0,05$. El modelo máximo de partida incluía todas las variables que habían resultado significativas en el análisis bivariante, a excepción de aquellas variables que parecen medir lo mismo como la escala HAD para las dimensiones ansiedad/depresión del EQ y reacción emocional del PSN, ejercicio para movilidad del EQ y movilidad física del PSN, AVD-B para cuidado personal del EQ y AVD-I para actividades cotidianas del EQ. Se calculó la razón de ventajas que correspondía a cada variable dentro del modelo multivariante. Para terminar se calculó mediante el coeficiente de correlación de Spearman la correlación entre el EAS y la puntuación obtenida en la EVA, así como una matriz de correlaciones entre las respectivas dimensiones del EQ y el PSN.

\section{RESULTADOS}

De las 2.002 cartas enviadas, 270 personas cumplieron criterios de exclusión, 453 rehusaron colaborar en el estudio, 49 estaban de viaje y otras 319 personas no pudieron ser localizadas tras tres intentos. Las 911 encuestas realizadas corresponden a un índice de respuesta de 66,8\% (IC: 64,2\%$69,3 \%$ ) calculado como el número de encuestas realizadas sobre el número de personas que cumplían criterios de inclusión y con las que se pudo entrar en contacto.

No se encontraron diferencias significativas en cuanto a distribución por sexo y edad entre el grupo de personas encuestadas y el grupo de personas que no se pudo encuestar por razones diferentes a cumplir algún criterio de exclusión.

La tabla 1 describe la muestra en cuanto a las variables que participan en el análisis posterior. La edad media de las personas de la muestra es de 74,7 años (IC: 74,3-75,1), son mujeres el 59,7\% (IC: 56,4-62,9), carecen de estudios (analfabetos o sólo leen y escriben) el 40,9\% (IC: 37,7-44,2), pertenecen a las clases sociales más favorecidas (I y II) el 38,3\% (IC: 35,1-41,6). Perciben su estado de salud como regular, malo o muy malo el 47,9\% (IC: 44,6 - 51,2), con mayor frecuencia las mujeres. El valor medio de la EVA es 66,6 (IC:65,3 - 68) y la mediana 70. El 48,8\% (IC: 45,5 - 52,1) de la muestra presenta valores en la EVA inferiores a la mediana, siendo esto más frecuente entre las mujeres. El 54,4\% (IC: 51,1 - 57,7) presentan un perfil de Euroqol distinto del óptimo (11111), ocurriendo con más frecuencia entre las mujeres y los mayores de 80 años. Los siguientes porcentajes de la muestra padecen problemas en las distintas dimensiones del Euroqol: 36,1\% (IC: 33- 39,3) en movilidad, 7,7\% (IC: 6,1-9,7) en cuidado personal, 19,9\% (IC: 17,4-22,7) en actividades cotidianas, 38,2\% (IC: 35-41,5) en dolor/malestar y $27 \%(24,1-30)$ en ansie$\mathrm{dad} /$ depresión. Las mujeres presentan más problemas que los hombres en todas las di- 
mensiones del Euroqol, excepto cuidado personal y los mayores de 80 años que los menores excepto en dolor/malestar y ansiedad/depresión. En el Perfil de Salud de Nottingham los siguientes porcentajes de la muestra puntúan más de 0 en las dimensiones: $36,1 \%$ (IC: 33-39,3) en energía, 63,4 (IC: 60,1-66,6) en dolor, 74,4\% (IC: 71,4-77,2) en movilidad física, 62,1 (IC: 58,9-65,3) en sueño, 71,3 (IC: 68,1-74,2) en reacción emocional y 33,3 (IC: $30,3-36,5)$ en aislamiento social. Las mujeres puntúan más de 0 con mayor frecuencia que los hombres en todas las dimensiones, y los mayores de 80 años en todas excepto sueño y reacción emocional. Para un análisis descriptivo más amplio ver Azpiazu y cols ${ }^{32}$.

Las variables tratadas como variables dependientes en el análisis multivariante (EAS: Muy Bueno o Bueno/ Regular, Malo o Muy malo; EVA: < 70/ $\geq 70$; Perfil de EQ $=11111 / \neq 11111$ y Existencia de Problemas en las dimensiones del EQ y PSN) se asocian en el análisis bivariante con casi todas las variables contempladas como se puede comprobar en la tabla 2.

Los factores que se asocian en el análisis multivariante con mala salud percibida, puntuación en la EVA inferior a 70 y perfil de EQ distinto del perfil «11111» se presentan en la tabla 3. La existencia de trastornos depresivos y de ansiedad son factores que se asocian intensamente a los tres instrumentos, también la falta de ejercicio y la dependencia para las AVD-I aparecen como factor asociado en los tres casos. El coeficiente de correlación de Spearman entre la puntuación de la EVA (de 0 a 100) y los cinco grados de EAS (muy malo, malo, regular, bueno, muy bueno) es $0,586(p<0,001)$ de forma que a mayor puntuación obtenida en la EVA, mejor EAS.
En la tabla 4 se encuentran los factores asociados a la presencia de problemas en cada una de las dimensiones del EQ. En el análisis multivariante se encuentra asociación de un grupo de variables con muchas de las dimensiones del EQ: la dependencia para las AVD-I aparece como factor asociado a todas las dimensiones; la presencia de trastornos depresivos se asocia con todas excepto cuidado personal y los trastornos de ansiedad con todas excepto actividades cotidianas, los problemas de vista se relacionan con todas excepto dolor/malestar, la dependencia para las AVD-B con todas excepto dolor/malestar y ansiedad/depresión, la falta de ejercicio se asocian con todas excepto dolor/malestar.

En la tabla 5 se representan los factores asociados a la presencia de problemas (puntuación superior a 0) en las distintas dimensiones del PSN. Son diferentes los conjuntos de variables que constituyen las ecuaciones de regresión logística múltiple para cada dimensión del PSN, pero hay un grupo de variables presentes en el modelo multivariante de casi todas las dimensiones. Estas variables comunes son las variables de salud mental y capacidad funcional. La presencia de trastornos de ansiedad se asocia con todas las dimensiones, y la de trastornos depresivos se asocia con todas menos dolor y sueño. Por otra parte la dependencia para las AVD-I y las AVD-B se asocia con todas las dimensiones a excepción de aislamiento social para AVD-I y sueño para las AVD-B. La falta de ejercicio también se asocia con todas excepto sueño.

En la tabla 6 se presenta una matriz de correlaciones entre las distintas dimensiones del EQ y del PSN. 


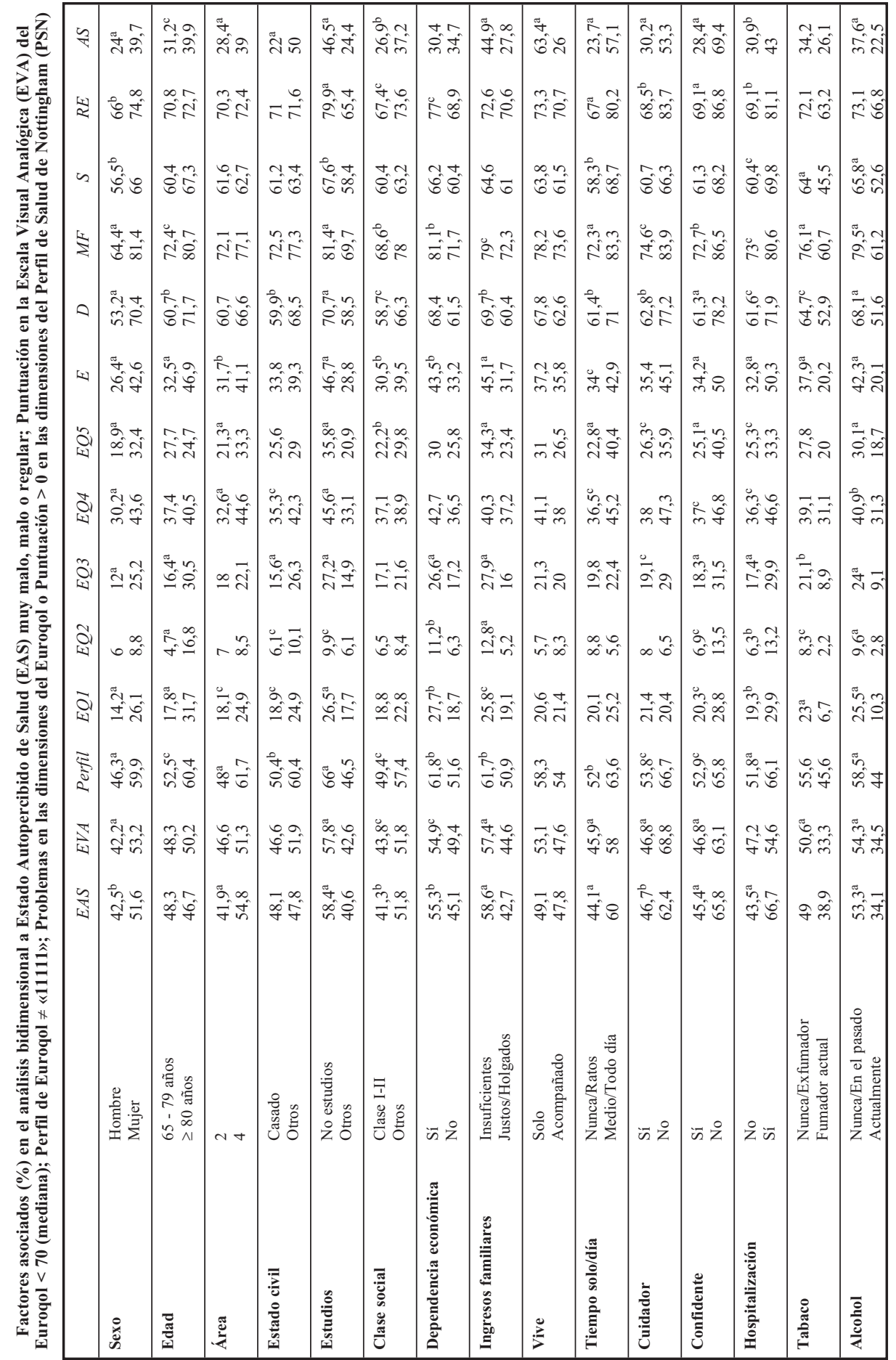




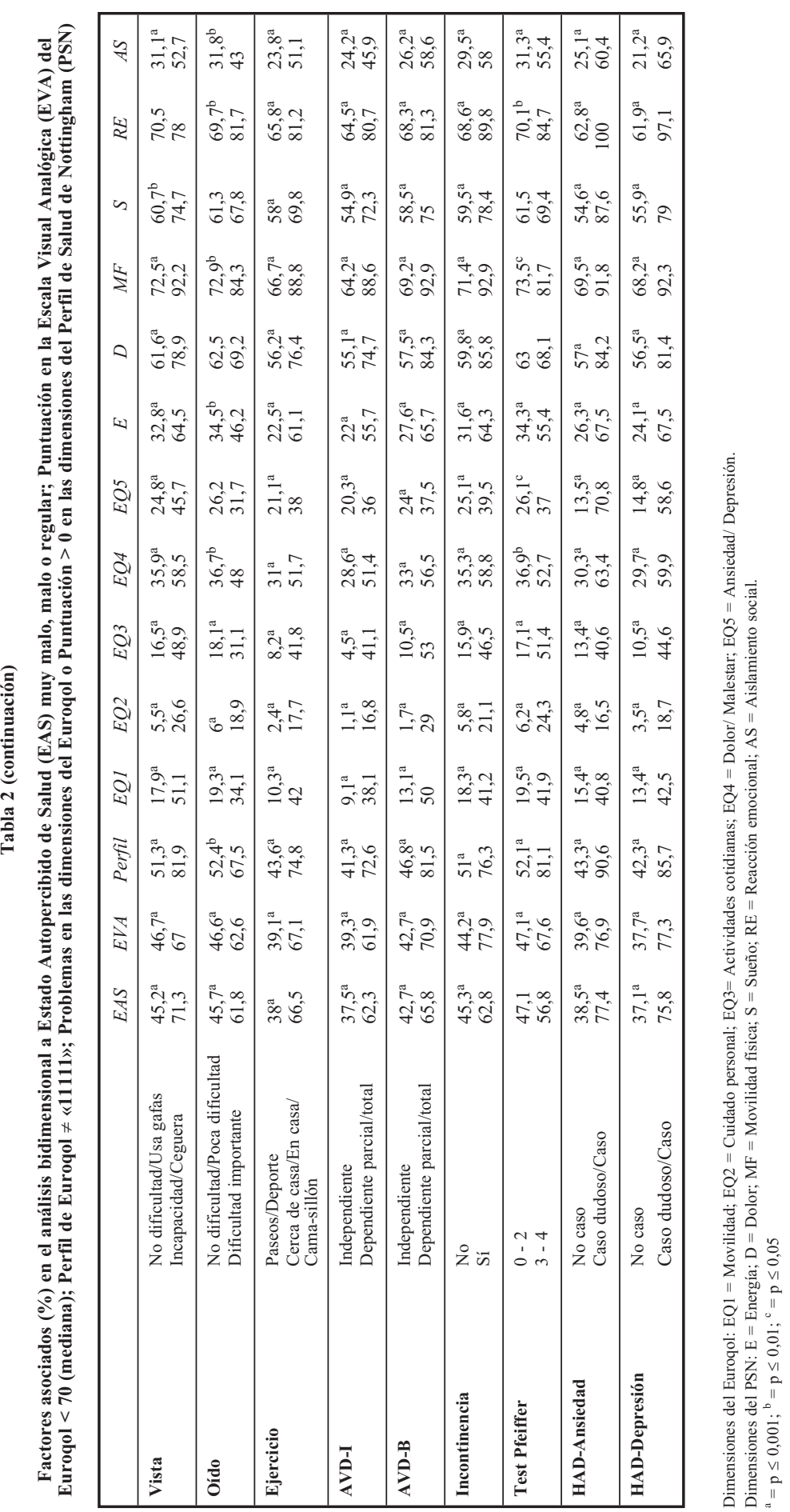


Tabla 3

Odd Ratios de los factores asociados mediante regresión logística múltiple a mala salud percibida y a mala calidad de vida

\begin{tabular}{|ccc|}
\hline Mala salud percibida & EVA $<70$ & Perfil $\neq$ «1111» \\
\hline Ansiedad & Ansiedad & Ansiedad \\
$4(2,6-6,2)$ & $2,8(1,8-4,3)$ & $7,9(4,5-13,9)$ \\
Depresión & Depresión & Depresión \\
$2(1,3-3)$ & $2,3(1,5-3,5)$ & $2,9(1,8-4,8)$ \\
Dependencia en AVD-I & Incontinencia & Dependencia en AVD-B \\
$1,7(1,2-2,4)$ & $3,1(1,8-5,4)$ & $2,5(1,5-4,1)$ \\
Falta de Ejercicio & Dependencia en AVD-I & Dependencia en AVD-I \\
$1,8(1,3-2,6)$ & $1,5(1,1-2,1)$ & $2(1,4-2,9)$ \\
Hospitalización & Falta de Ejercicio & Falta de Ejercicio \\
$2,1(1,4-3,1)$ & $1,8(1,3-2,6)$ & $1,8(1,2-2,7)$ \\
Falta de Confidente & Falta de Cuidador & Incapacidad o Ceguera \\
$2,3(1,4-4,1)$ & $2,3(1,4-3,8)$ & $0,5(0,2-0,9)$ \\
Area 4 & Bebe alcohol & Area 4 \\
$1,8(1,3-2,5)$ & $0,6(0,4-0,9)$ & $1,9(1,3-2,6)$ \\
Bebe alcohol & & \\
$0,7(0,5-1)$ & & \\
\hline
\end{tabular}

Tabla 4

Odd Ratios de los factores asociados mediante regresión logística múltiple a la existencia de problemas en las dimensiones del Euroqol

\begin{tabular}{|ccccc|}
\hline Movilidad & Cuidado personal & Actividades cotidianas & Dolor/Malestar & Ansiedad/Depresión \\
\hline Dependencia en AVD-B & Dependencia en AVD-I & Dependencia en AVD-B & Ansiedad & Solo todo ó medio día \\
$4,8(2,7-8,5)$ & $7,1(2,9-17,2)$ & $4,8(3-7,6)$ & $2,7(1,9-3,9)$ & $1,8(1,2-2,6)$ \\
Dependencia en AVD-I & Falta de Ejercicio & Falta de Ejercicio & Dependencia en AVD-I & Incapacidad ó Ceguera \\
$2,9(1,9-4,5)$ & $3,6(1,9-7)$ & $3,9(2,5-6,2)$ & $1,9(1,4-2,6)$ & $1,7(1-2,8)$ \\
Incapacidad ó Ceguera & Edad $\geq 80$ años & Pfeiffer $3-4$ & Depresión & Área 4 \\
$2,2(1,3-3,7)$ & $2,4(1,3-4,4)$ & $3,5(1,8-6,7)$ & $1,8(1,3-2,6)$ & $1,7(1,2-2,4)$ \\
Depresión & Incapacidad ó Ceguera & Depresión & Area 4 & Falta Confidente \\
$1,8(1,2-2,8)$ & $2,2(1,1-4,2)$ & $3,1(2-4,9)$ & $1,7(1,3-2,3)$ & $1,7(1-2,8)$ \\
Ansiedad & Ansiedad & Incapacidad ó Ceguera & Incontinencia & Mujer \\
$1,8(1,2-2,8)$ & $2,2(1,2-3,9)$ & $2,2(1,2-4)$ & $1,7(1,1-2,7)$ & $1,6(1,1-2,4)$ \\
Fumador & Ingr. fam. Insuficientes & Falta de cuidador & & Dependencia en AVD-I \\
$0,4(0,1-0,9)$ & $2,1(1,2-3,8)$ & $2,1(1,1-4)$ & & $1,6(1,1-2,3)$ \\
Incontinencia & Mala audición & Dependencia económica & & No estudios \\
$0,4(0,2-0,8)$ & $1,9(1-3,6)$ & $1,8(1,1-2,9)$ & & $1,5(1,1-2,2)$ \\
Toma Alcohol & & No Casado & & Falta de Ejercicio \\
$0,6(0,3-1)$ & & $1,7(1,1-2,7)$ & & $1,4(1-2,1)$ \\
\hline
\end{tabular}


Tabla 5

Odd Ratios de los factores asociados mediante regresión logística múltiple a puntuación $>0$ en las dimensiones del Perfil de Salud de Nottingham

\begin{tabular}{|cccccc|}
\hline Energia & Dolor & Movilidad fisica & Sueño & Reac. emocional & Aislamiento social \\
\hline Ansiedad & Dependencia AVD-B & Dependencia AVD-B & Ansiedad & Incontinencia & Vive solo \\
$3(2-4,6)$ & $2,9(1,8-4,7)$ & $3,4(1,8-6,4)$ & $5,3(3,4-8,2)$ & $4,4(1,9-10,1)$ & $4,9(2,7-8,8)$ \\
Falta de Ejercicio & Ansiedad & Dependencia AVD-I & Dependencia AVD-I & Falta de Confidente & Depresión \\
$2,8(1,9-4)$ & $2,3(1,5-3,6)$ & $2,8(1,9-4,3)$ & $1,8(1,3-2,4)$ & $3,1(1,5-6,3)$ & $3,3(2,1-5,1)$ \\
Depresión & Falta de Cuidador & Depresión & Fumador & Dependencia AVD-I & Falta de Confidente \\
$2,5(1,7-3,7)$ & $2(1,2-3,5)$ & $2,5(1,4-4,4)$ & $0,6(0,3-0,9)$ & $2,1(1,4-3,1)$ & $2,8(1,5-5,4)$ \\
Dependencia AVD-I & Dependencia AVD-I & Ansiedad & & Sin Estudios & Ansiedad \\
$2,3(1,6-3,4)$ & $1,6(1,1-2,3)$ & $2,3(1,3-4,1)$ & & $2(1,4-2,9)$ & $2,4(1,5-3,8)$ \\
Dependencia AVD-B & Falta de Ejercicio & Mujer & & Hospitalización & Dependencia AVD-B \\
$1,9(1,2-3)$ & $1,6(1,1-2,3)$ & $1,7(1,2-2,4)$ & & $1,7(1,1-2,6)$ & $2,4(1,5-3,8)$ \\
Area 4 & Mujer & Toma Alcohol & & Falta de Ejercicio & Area 4 \\
$1,6(1,1-2,3)$ & $1,5(1,1-2,1)$ & $0,6(0,4-0,9)$ & & $1,6(1,1-2,4)$ & $2(1,3-3)$ \\
Toma Alchol & & & Solo medio ó todo día & Falta de Cuidador \\
$0,6(0,4-0,9)$ & & & $1,5(1-2,2)$ & $2(1,1-3,7)$ \\
& & & Dependencia AVD-B & Sin Estudios \\
& & & & $0,5(0,3-0,9)$ & $1,9(1,3-2,9)$ \\
& & & & Falta de Ejercicio \\
& & & & & $1,9(1,3-2,9)$ \\
& & & & & No Casado \\
& & & & & $1,6(1-2,6)$ \\
\hline
\end{tabular}

Tabla 6

Matriz de correlaciones entre las dimensiones del Euroqol y del Perfil de Salud de Nottingham

\begin{tabular}{|l|lcccccc|}
\hline \multicolumn{2}{|c}{} & \multicolumn{7}{c|}{ Perfil de Salud de Nottingham } \\
\cline { 3 - 8 } & & Energía & Dolor & $\begin{array}{c}\text { Movilidad } \\
\text { fisica }\end{array}$ & Sueño & $\begin{array}{c}\text { Reacción } \\
\text { emocional }\end{array}$ & $\begin{array}{c}\text { Aislamiento } \\
\text { social }\end{array}$ \\
\hline & Movilidad & 0,517 & 0,458 & 0,546 & 0,170 & 0,335 & 0,277 \\
$\mathbf{E}$ & Cuidado personal & 0,362 & 0,228 & 0,369 & 0,131 & 0,224 & 0,287 \\
$\mathbf{R}$ & Actividades cotidianas & 0,506 & 0,373 & 0,507 & 0,239 & 0,363 & 0,338 \\
$\mathbf{Q}$ & Dolor/malestar & 0,458 & 0,562 & 0,422 & 0,301 & 0,363 & 0,237 \\
$\mathbf{O}$ & Ansiedad/Depresión & 0,353 & 0,247 & 0,289 & 0,354 & 0,569 & 0,422 \\
\hline
\end{tabular}

Todos los coeficientes de correlación de Spearman son significativos con una $\mathrm{p}<0,01$

\section{DISCUSIÓN}

Otros autores ${ }^{22,28,33,34}$ han estudiado con anterioridad qué factores se asocian al estado de salud autopercibido, sin embargo nuestro trabajo incluye el estudio de variables de estado de ánimo y clase social todavía poco estudiadas, y aporta también el estudio de los factores asociados a dos instrumentos de medida de la calidad de vida relacionada con la salud, como son el Euroqol y el Perfil de Salud de Nottingham.
Algunas de estas investigaciones del estado autopercibido de salud ${ }^{33,34}$ incluyen dentro de su población de estudio personas con deterioro cognitivo importante, recogiendo la información referente a estas personas a partir de sus cuidadores. En nuestro trabajo hemos excluido a las personas que presentaban un deterioro cognitivo moderado o severo, porque pensamos que no sería fiable la información obtenida al encuestarles y tampoco nos pareció válida la información obtenida de sus cuidadores, pues lo que buscá- 
bamos era conocer la apreciación subjetiva de la persona seleccionada. Creemos que sería necesario investigar y poner en marcha otros modos de acceder a la subjetividad de las personas que no pueden expresarse verbalmente por sufrir un deterioro cognitivo, pero éste no es el objetivo de nuestro estudio.

El porcentaje de respuesta de la encuesta ha sido del $66,8 \%$, algo inferior a los índices obtenidos en otros estudios de características similares, como «Envejecer en Leganés $»^{35}(80 \%)$ y el estudio ANCO $^{36}(88 \%)$ que mejoraron su índice de respuesta mediante una campaña informativa en prensa, televisión y radio. No obstante creemos que las respuestas obtenidas por nosotros son representativas de la población de referencia, pues no ha habido diferencias significativas en cuanto a edad y sexo entre la población encuestada y la que no se logró encuestar.

Prácticamente todos los factores estudiados se asocian en el análisis bivariado a la mayoría de las variables dependientes (EAS, EVA, Perfil y dimensiones del Euroqol y dimensiones del PSN), a excepción de vivir solo o acompañado, sin embargo sí se asocia pasar o no gran parte del día solo.

En el análisis multivariado los factores que se asocian casi constantemente con la detección de problemas de la calidad de vida o del estado autopercibido de salud son la presencia de trastornos de ansiedad y/o depresión, la falta de ejercicio y la dependencia para las actividades básicas y/o instrumentales de la vida diaria.

Se ha optado por dicotomizar todas las variables independientes para facilitar la interpretación de los resultados, aunque somos conscientes de que esto puede enmascarar o modificar algunas asociaciones.

Teniendo en cuenta que nuestro trabajo es un corte transversal en el que no se puede determinar causalidad, los resultados obtenidos tanto en el análisis bivariado como en el multivariado del estado autopercibido de salud encajan dentro del modelo de estructura del estado de salud propugnado por Johnson y Wolinsky ${ }^{3}$. La falta de ejercicio se ha investigado mediante la pregunta «¿Hace Ud. algo de ejercicio?», a la que la persona encuestada puede contestar «Permanezco todo el día en la cama o sentado», «Me muevo sólo por la casa», «Salgo a la calle en las cercanías de mi casa», «Paseo una hora o más todos o casi todos los días», «Practico algún deporte (natación, gimnasia, bicicleta, bolos, petanca...)», y con esta pregunta se mide la actividad física realizada, aunque posiblemente en la mayor parte de las personas que han contestado las dos primeras respuestas concurran una falta de ejercicio con una incapacidad para la movilidad (déficit físico). La capacidad para realizar actividades de la vida diaria básicas e instrumentales, investigada mediante un instrumento que pide a la persona encuestada que valore su capacidad para llevar a cabo ciertas actividades necesarias para vivir independientemente, se refiere a una limitación funcional que puede conducir a una limitación social. Beland y Zunzunegui modifican el modelo de Johnson y Wolinsky, dándole influencia a los trastornos depresivos sobre los distintos elementos del modelo de estructura del estado de salud. En nuestro estudio los trastornos de ansiedad también aparecen como factores asociados con la mayoría de las variables dependientes.

Las asociaciones con un mal estado de salud autopercibido encontradas en nuestro análisis bivariante van, en general, en la misma dirección que las descritas en la bibliografía en mayores de 65 años: bajos ingresos económicos ${ }^{28,37}$, sentimiento de sole$\mathrm{dad}^{28}$, problemas visuales ${ }^{28}$, estilo de vida sedentario $^{28}$, incapacidad funcional ${ }^{8,33,34}$, bajo nivel de estudios ${ }^{22}$, falta de apoyo emocional $^{22}$, falta de apoyo social ${ }^{22,37}$ y sexo femenino ${ }^{37}$.

Algunas de las variables que resultan significativas en nuestro análisis multivariante del estado autopercibido de salud también lo 
son en los análisis multivariantes realizados por otros autores ${ }^{29,35,38-40}$, aunque en ninguno de estos estudios consultados se parte del mismo conjunto de variables del que parte este estudio. Concretamente, las variables referidas al estado de ánimo de las personas encuestadas (ansiedad y depresión) no son exploradas por casi ninguno de estos estudios siendo, sin embargo, éste un aspecto que la gente considera muy importante y de hecho en nuestro estudio estas variables tienen una asociación muy fuerte con el estado autopercibido de salud.

Béland y Zunzuneguii ${ }^{29,38}$ en su modelo de regresión logística múltiple con 896 personas mayores de 65 años de Leganés encuentran asociación del estado de salud autopercibido malo y regular con respecto a bueno con una menor edad, presencia de enfermedades crónicas, invalidez, problemas de visión, dependencia parcial para las AVD-I y presencia de sintomatología depresiva.

Ruigómez y cols ${ }^{39}$ en la Encuesta de Salud de Barcelona de 1986 ( $\mathrm{n}=1.288)$ obtienen asociación en el análisis multivariante de la percepción por parte de los mayores de 65 años de su estado de salud como malo con ser mujer, pertenecer a las clases sociales III, IV o V, presentar dos o más trastornos crónicos, ser dependiente para las actividades básicas de la vida diaria, haber tenido restringida la actividad en las semanas anteriores a la entrevista y ser sedentario.

En el estudio de Ferrando y cols ${ }^{22}$, realizado con datos de la Encuesta de Salud de Barcelona de 1992 ( $n=1.156)$, el mal estado percibido de salud en las mujeres mayores de 60 años se relaciona con la necesidad de apoyo para la vida cotidiana y el tener un nivel educativo de estudios primarios incompletos, y en los hombres mayores de 60 años con la ausencia de apoyo emocional y la necesidad de apoyo en el cuidado personal.

Otros autores,27 encuentran que padecer enfermedades ${ }^{27,28,40}$, consumir medicamen$\operatorname{tos}^{27,28,40}$, perder capacidad funcional $27,28,40,41$, insomnio $^{41}$, insatisfacción con la propia vi $\mathrm{da}^{41}$, pertenecer a una clase social más desfavorecida $^{40}$ y ser mujer ${ }^{40}$ se relacionan con una peor percepción por parte de los ancianos del propio estado de salud.

Existe poca bibliografía que explore qué factores se asocian a mala calidad de vida en el Euroqol, y la que hay se refiere a población general, y no concretamente a población anciana. Badia y cols ${ }^{42,43}$ analizan los datos de la Encuesta catalana de salud de 1994 en la que se incluyó el Euroqol en las encuestas dirigidas a los mayores de 15 años. Kind y cols ${ }^{44}$ analizan los datos de una encuesta poblacional en el Reino Unido, realizada en 1993 a mayores de 18 años que viven en la comunidad. Ninguno de estos autores realiza un análisis multivariante.

No hemos encontrado estudios que analicen en población anciana mediante análisis multivariante los factores asociados a mala calidad de vida en las dimensiones del PSN. Los trabajos consultados ${ }^{45,46}$ hacen únicamente análisis bivariante, encontrando, al igual que nosotros, asociación de mayor puntuación en diversas dimensiones del PSN con el sexo femenino (todas excepto energía $^{45}$; todas ${ }^{46}$ ), mayor edad (todas excepto dolor y sueño ${ }^{45}$; sólo movilidad ${ }^{46}$ ), menor nivel educativo (energía, dolor y movilidad $^{45}$ ), dependencia para las AVD-B (todas excepto sueño ${ }^{46}$ ), deterioro cognitivo (dolor, reacción emocional y aislamiento social $\left.^{46}\right)$. Mientras que nuestro estudio encuentra únicamente asociación de vivir sólo con mayor porcentaje de problemas en la dimensión aislamiento social, Bayó y cols $^{46}$ obtienen también mayor puntuación en las dimensiones dolor y reacción emocional. Por otra parte la puntuación de todas las dimensiones excepto dolor y sueño en el estudio de Casado y cols. ${ }^{45}$ es mayor en las personas que no viven en pareja que en las que sí lo hacen. Otras variables asociadas en estos estudios a una mayor puntuación en algunas dimensiones del PSN son el uso de recursos sociales (sueño, aislamiento social y movilidad $)^{46}$ y el grado de morbilidad: ma- 
yor número de patologías crónicas (todas las dimensiones ${ }^{45}$ sólo dolor ${ }^{46}$ ), mayor consumo de fármacos (todas las dimensiones) ${ }^{45} \mathrm{e}$ inclusión en el programa de atención domiciliaria (todas excepto dolor) ${ }^{45}$.

Bucquet y Curtis ${ }^{47}$ en un estudio con población general mayor de 16 años de tres barrios londinenses buscan si hay diferencias en las puntuaciones de las distintas dimensiones al variar edad, sexo y clase social (clases de trabajadores manuales y no manuales). Estos autores obtienen mediante regresión logística multivariante asociación del sexo con todas las dimensiones excepto aislamiento social; de la edad con todas excepto reacción emocional; y de la clase social con energía, dolor, reacción emocional y sueño. El presente estudio obtiene asociación de la variable sexo sólo con dolor y movilidad física; la variable edad no se asocia con ninguna de las dimensiones, aunque hay que tener en cuenta que el rango de edad es muy diferente del rango del estudio de Bucquet y Curtis; por último, a pesar de que la variable clase social no se asocia con ninguna de las dimensiones en nuestros resultados sí lo hace con reacción emocional otra variable indicadora de nivel socioeconómico, ingresos familiares justos.

La presencia de asociación de distintas dimensiones de un mismo instrumento de medida de la calidad de vida ( Euroqol o PSN) con las mismas variables (trastornos depresivos y de ansiedad, dependencia para las actividades básicas y/o instrumentales de la vida diaria, ejercicio físico, trastornos visuales) está de acuerdo con el concepto multifactorial de salud, en el que los distintos factores (físicos, psíquicos y sociales) no pueden ser perfectamente delimitados, sino que se hallan interrelacionados unos con otros influyéndose mutuamente.

En nuestro estudio hemos utilizado tres medidas del estado de salud, de las cuales dos de ellas (el estado autopercibido de salud y El Euroqol (EVA) ofrecen una valoración general mientras que las dimensiones del Euroqol y del PSN entran a analizar los componentes de la calidad de vida relacionada con la salud.

Existiendo correlación entre el EAS y la EVA, la pregunta de la Encuesta Nacional de Salud «¿Cómo describiría su estado de salud en la actualidad?» permite sólo 5 posibles respuestas (muy bueno, bueno, regular, malo y muy malo), mientras que la EVA permite una mayor amplitud de respuestas. Los factores asociados con estado de salud autopercibido regular, malo o muy malo, puntuación en la EVA menor de 70 y perfil de Euroqol $\neq 11111$ mayormente son los mismos y en los tres casos presentan Odd Ratios de valor similar. Parece ser que estos tres instrumentos generales están midiendo más o menos lo mismo. Únicamente es sorprendente que beber alcohol actualmente es un factor protector frente a un mal estado de salud autopercibido y una puntuación en la EVA baja, lo cual posiblemente podría explicarse porque las personas que toman alcohol a edad avanzada es porque se encuentran bien de salud, pues si no ya lo hubieran dejado anteriormente. También padecer ceguera o incapacidad visual importante inexplicablemente aparece como factor protector frente a presentar un Perfil de Euroqol distinto del óptimo, asociándose sin embargo con mayor frecuencia de problemas en la mayoría de las dimensiones del Euroqol.

Existe correlación entre las dimensiones equivalentes del Euroqol (movilidad, dolor/malestar y ansiedad/depresión) y del PSN (movilidad física, dolor y reacción emocional), no habiendo diferencias importantes en el análisis multivariante en cuanto a los factores que se asocian a la existencia de problemas en las dimensiones equivalentes de cada uno de los cuestionarios. La principal diferencia que encontramos es la mayor importancia de los problemas sensoriales en el Euroqol (trastornos visuales en todas las dimensiones excepto dolor/ malestar y trastornos auditivos en cuidado personal) frente al PSN, en el que no aparecen los problemas sensoriales en ninguna dimensión. 
Tendría interés la inclusión de un instrumento genérico de calidad de vida en los estudios de salud poblacionales que nos orientase sobre las dimensiones mayormente deficitarias en un momento dado y su evolución posterior en el tiempo bajo la influencia de las políticas de salud, que a la vista de nuestros resultados deben incluir elementos que ayuden a las personas mayores a conservar su autonomía durante más tiempo, especialmente en lo referente a su capacidad funcional y su salud mental.

Después de analizar los tres instrumentos de medida del estado de salud, creemos que el Euroqol tiene ventajas frente a los otros dos. Además de permitir una mayor amplitud de respuestas a la valoración global del estado de salud, hace una somera valoración de algunos de los factores más importantes para la calidad de vida, dando una mayor información que el estado de salud autopercibido, siendo la inversión adicional de tiempo pequeña. El PSN necesita un mayor número de preguntas (8 ítems para las dimensiones movilidad y dolor/malestar y 9 para la dimensión reacción emocional) para conseguir la información que el Euroqol consigue con una única pregunta, siendo más o menos los mismos factores los asociados a la presencia de problemas en las dimensiones equivalentes. Por otra parte el PSN no ofrece una valoración global del estado de salud.

\section{BIBLIOGRAFÍA}

1. Badia X, Patrick KD. La medida de la salud en la investigación y práctica clínica. Mahón. Escuela de verano de Salud Pública; 1995.

2. Lizán Tudela L. Enfoque genérico de la calidad de vida desde el punto de vista de la psicología y de la medicina de familia. Concepto de calidad de vida y sus dimensiones. Aten Primaria 1995; 16 (Suplem 1): 131-132.

3. Johnson RJ, Wolinsky FD. The structure of health status among older adults: Disease, disability, functional limitation and perceived health. J Health Soc Behav 1993; 34: 105-121.
4. Verbrugge LM, Jette AM. The disablement process. Soc Sci Med 1994; 38 (1): 1-14.

5. Fletcher A, Dickinson EJ, Philp I. Review: audit measures: Quality of life instruments for everyday use with elderly patients. Age Ageing 1992; 21: $142-150$

6. Alvarez Solar M. Coordinación asistencial en la atención al anciano. Aten Primaria 1995; 16 (Suplem 1): 117-120.

7. Insalud. Jornadas europeas sobre atención a las personas mayores dependientes: la respuesta sociosanitaria. Madrid;1995.

8. Inserso. Plan Gerontológico. Madrid: Inserso; 1993.

9. Ministerio de Asuntos Sociales y Ministerio de Sanidad y Consumo. Acuerdo marco entre los Ministerios de Asuntos Sociales y Sanidad y Consumo en materia de coordinación socio-sanitaria. 14 de diciembre de 1993. Madrid: Ministerio de Asuntos Sociales y Ministerio de Sanidad y Consumo; 1993.

10. Ministerio de Asuntos Sociales y Ministerio de Sanidad y Consumo. Convenio de colaboración interministerial para la atención socio-sanitaria de las personas mayores. 14 de diciembre de 1993. Madrid: Ministerio de Asuntos Sociales y Ministerio de Sanidad y Consumo; 1993.

11. Insalud. Criterios de ordenación de servicios para la atención sanitaria a las personas mayores. Madrid: Insalud; 1996.

12. García Cardova F. Políticas sanitarias y sociales desarrolladas en algunos países de la Comunidad Europea con respecto a la tercera edad. Aten Primaria 1994; 13 (9): 507-14.

13. Benítez Rosario MA, Vázquez Díaz JR. Valoración del grado de autonomía de los ancianos. Aten Primaria 1992; 10 (7): 888-91.

14. Béland F, Zunzunegui V. Presentación del estudio «Envejecer en Leganés». Rev Gerontol 1995; 5: 207-14.

15. Farquhar M. Elderly people's definitions of quality of life. Soc Sci Med 1995; 41 (10): 1439-46.

16. Rubio Herrera R, Aleixandre Rico M, Villaverde C, Cabezas Casado JL, Castellón A. Calidad de vida y senectud, estudio comparativo en función del sexo. Geriatrika 1997; 13: 383-8.

17. Rubio Herrera R, Aleixandre Rico M, Cabezas Casado JL. Estudio sobre la valoración de la cali- 
dad de vida en la población anciana andaluza. Geriatrika 1997; 13: 271-81.

18. Raphael D, Brown I, Renwick R, Cava M, Weir $\mathrm{N}$, Heathcote $\mathrm{K}$. The quality of life of seniors living in the community: a conceptualization with implications for public health practice. Can J Public Health 1995; 86 (4): 228-33.

19. Raphael D, Brown I, Renwick R, Cava M, Weir N, Heathcote K. Measuring quality of life of older persons: a model with implications for community and public health nursing. Int J Nurs Stud 1997; 34 (3): 231-39.

20. Kerschner H, Pegnes JAM. Productive aging: A quality of life agenda. J Am Diet Assoc 1998; 98 : $1445-8$

21. Lapierre S, Bouffard L, Bastin E. Personal goals and subjective well-being in later life. Int J Aging Hum Dev 1997; 45 (4): 287-303.

22. Ferrando J, Nebot M, Borrell C, Egea L. Apoyo social y estado de salud percibido en población no institucionalizada de más de 60 años. Gac Sanit 1996; 10: 174-182.

23. García Ameijeiras C, Roigé Cortadelles T, Elías Aldosa T, Viñas Folch P. Utilidad de un instrumento de valoración gerontológica multidisciplinar en la asignación de recursos socisanitarios. Aten Primaria 1997; 20 (9): 506-10.

24. Azpiazu Garrido M. Calidad de vida y factores asociados en mayores de 65 años. [Tesis doctoral]. Madrid: Universidad Autónoma de Madrid; 2001 .

25. Ministerio de Sanidad y Consumo. Encuesta Nacional de Salud,1993. Madrid: Ministerio de Sanidad y Consumo; 1994

26. Badia X, Salamero M, Alonso J, Ollé A. La medida de la salud. Guía de escalas de medición en español. 2. ${ }^{a}$ ed. Barcelona: Edimac;1999.

27. Gómez Peligros A, Alonso Atienza MC, Menéndez Obregón JL, Chércoles Ruiz EM. Salud percibida en los ancianos de la provincia de Toledo y su relación con variables sanitarias. SEMER 1993; 19: 595-9.

28. Gorroñogoitia Iturbe A, Ibáñez Pérez F, Olaskoaga Arrate A. Autopercepción de salud en el anciano: relación con algunas variables socioeconómicas y de salud. Aten Primaria 1992; 10 (5): 771-6.

29. Béland F, Zunzunegui MV. La salud y las incapacidades funcionales. Elaboración de un modelo causal. Rev Gerontol 1995; 5: 259-73.
30. Herdman M, Badia X, Berra S. El Euroqol-5D: una alternativa sencilla para la medición de la calidad de vida relacionada con la salud en atención primaria. Aten Primaria 2001; 28 (6): 425-9.

31. The European Group for Quality of Life and Health Measurement. European guide to the Nottingham Health Profile. 1989. Revised edition.

32. Azpiazu Garrido M, Cruz Jentoft A, Villagrasa Ferrer JR, Abanades Herranz JC, García Marín $\mathrm{N}$, Alvarez de Mon Rego C. Calidad de vida en mayores de 65 años no institucionalizados de dos áreas sanitarias de Madrid. Aten Primaria (en prensa).

33. Espejo Espejo J, Martínez de la Iglesia J, Aranda Lara JM, Rubio Cuadrado V, Enciso Bergé I, Zunzunegui Pastor MV et al. Capacidad funcional en mayores de 60 años y factores sociosanitarios asociados (proyecto ANCO). Aten Primaria 1997; 20 (1): 3-11.

34. Ruigómez A, Alonso J. Validez de la medida de la capacidad funcional a través de las actividades básicas de la vida diaria en población anciana. Rev Gerontol 1996; 6: 215-23.

35. León Galat V, Zunzunegui MV, Béland F. El diseño y la ejecución de la encuesta «Envejecer en Leganés». Rev Gerontol 1995; 5: 215-31.

36. Martínez de la Iglesia J, Pérula de Torres LA Espejo J, Rubio V, Aranda JM, Fonseca FJ et al. Proyecto ANCO: un estudio sociosanitario de la población mayor de la ciudad de Córdoba. Diseño y ejecución. Rev Gerontol 1997; 7: 82-90.

37. Fernández Merino MC, Vérez Vivero L, Gude Sampedro F. Morbilidad crónica y autopercepción de salud en los ancianos de una comunidad rural. Aten Primaria 1996; 17 (2): 108-112.

38. Zunzunegui V, Béland F. La salud de las personas mayores de Leganés. Rev Gerontol 1995; 5 $245-58$

39. Ruigómez A, Alonso J, Antó JM. Salud percibida y capacidad funcional de la población anciana no institucionalizada de Barcelona. Gac Sanit 1991; 5 (24): 117-24.

40. Séculi E, Fusté J, Brugulat P, Juncá S, Rué M Guillén M. Percepción del estado de salud en varones y mujeres en las últimas etapas de la vida. Gac Sanit 2001; 15 (3): 217-23.

41. Lindgren A-M, Svärdsudd K, Tibblin G. Factors related to perceived health among elderly people: the Albertina project. Age Ageing 1994; 23: 328-33.

Rev Esp Salud Pública 2002, Vol. 76, N. ${ }^{\circ} 6$ 
42. Badia X, Schiaffino A, Alonso J, Herdman M. Using the Euroqol 5-D in the Catalan general population: feasibility and construct validity. Qual Life Res 1998; 7: 311-22.

43. Badia X, Fernández E, Segura A. Influence of socio-demographic and health status variables on evaluation of health states in a spanish population. European Journal of Public Health 1995; 5: $82-93$.

44. Kind P, Dolan P, Gudex C, Williams A. Variations in population health status: results from a United Kingdom national questionnaire survey. BMJ 1998; 316: 736-41.
45. Casado JM, González N, Moraleda S, Orueta R, Carmona J, Gómez-Calcerrada RM. Calidad de vida relacionada con la salud en pacientes ancianos en atención primaria. Aten Primaria 2001; 28 (3): 167-74.

46. Bayó J, Fernández-Aramburu MC, Orfila F, Dalfó A, Casajuana J, Vila MA et al. Autopercepción de salud y evaluación integral del paciente anciano en un centro de atención primaria. Aten Primaria $1996 ; 17$ (4): 273-9.

47. Bucquet D, Curtis S. Socio-demographic variation in perceived illness and the use of primary care service planning. Soc Sci Med 1986; 23 (7): 737-744 